\title{
CONVERGENCE OF CIRCLE PACKINGS OF FINITE VALENCE TO RIEMANN MAPPINGS
}

\author{
ZHENG-XU HE AND BURT RODIN
}

\begin{abstract}
In [R-S], the conjecture by W. Thurston [Th] that the hexagonal circle packings can be used to approximate the Riemann mapping (in the topology of uniform convergence in compact subsets) is proved; and in [He], the derivatives of these approximations are shown to be convergent.

We show in Section 1 that the methods used in [R-S] in the case of hexagonal packings can be easily extended to the case of nonhexagonal circle packing with bounded radii ratios. We note that Stephenson had taken the major steps toward such an extension in [Ste]. Although he follows the overall strategy of [R-S], he replaces certain key steps by parabolistic arguments which have an interesting nterpretation in terms of the flow of electricity in a network.

In Section 2, we show that the method of [He] can be extended to a more general class of non hexagonal packings. Specifically, the restriction in [Ste] that the radii ratios be bounded can be replaced by the much weaker condition that the circle packings have uniformly bounded valence.
\end{abstract}

\section{INTRODUCTION}

Let $P$ be a circle packing which "almost" fills up some fixed simply connected domain $\Omega$. Suppose that the nerve (or graph) of $P$ is equivalent to the 1-skeleton of a triangulation of the closed unit disk $\bar{D}=\{z \in \mathbb{C} ;|z| \leq \mid 1\}$. Then by the Circle Packing Theorem, there is a circle packing $P^{\prime}$ in $\bar{D}$ which is combinatorially equivalent to $P$, such that all the boundary circles of $P^{\prime}$ are tangent to the unit circle. Let $\Omega_{P}$ be the union of all triangles of centers of mutually tangent triples of circles in $P$. As in [R-S], there is a simplicial map $f_{P}: \Omega_{P} \rightarrow D$ which maps the centers of circles of $P$ into the centers of circles 
of $P^{\prime}$. We may normalize $f_{P}$ by a Möbius transformation so that

$$
f_{P}\left(z_{0}\right)=0, f_{P}\left(z_{1}\right)>0
$$

for some pre-assigned points $z_{0}, z_{1} \in \Omega$. Note that if $\partial \Omega_{P}$ is sufficiently close to $\partial \Omega$, then $z_{0}, z_{1} \in \Omega_{P}$.

Let $f: \Omega \rightarrow D$ be the Riemann mapping with

$$
f\left(z_{0}\right)=0, f\left(z_{1}\right)>0
$$

Suppose that both the Caratheodory distance

$$
d\left(\partial \Omega_{P}, \partial \Omega\right)=\max \left\{\sup _{w \in \partial \Omega} \inf _{z \in \partial \Omega_{P}}|z-w|, \sup _{w \in \partial \Omega_{P}} \inf _{z \in \partial \Omega}|z-w|\right\}
$$

and the mesh,

$$
m(P)=\max \{\text { radii of circles of } P\}
$$

are small, say, $\leq \epsilon$. We are interested in the the following problem: How close is $f_{P}$ to the Riemann mapping $f$ in terms of $\epsilon$ ?

In [R-S], it is shown that if the packings $P$ are subpackings of regular hexagonal circle packings, then in any compact subset $K \subseteq \Omega$,

$$
\left\|f_{P}-f\right\|_{K} \rightarrow 0 \text { as } \epsilon \rightarrow 0
$$

where $\|\cdot\|_{K}$ denotes $L^{\infty}(K)$ - norm. Then in [He], it is further shown that

$$
\left\|\frac{\partial f_{P}(z)}{\partial z}-\frac{\partial f(z)}{\partial z}\right\|_{K} \rightarrow 0 \text { as } \epsilon \rightarrow 0 \text {. }
$$

It follows from (0.4) that $\left\|\frac{\partial f_{P}(z)}{\partial \bar{z}}\right\|_{K} \rightarrow 0$ and $\left\|r_{P}-\left|f^{\prime}\right|\right\|_{K} \rightarrow 0$, where $r_{P}$ is the ratio of the radii of a target circle to its source circle nearest to $z$ (see [Ro]).

In this paper, we will show that the results of [R-S] and [He] can be generalized easily for more general circle packings. The generalized approach of [He] leads to the following result. 
Theorem 2.1. Assume that the valences of $P$ are bounded by a positive integer $k_{0}$. Let $K \subseteq \Omega$ be a compact subset. Then

$$
\left\|f_{P}-f\right\|_{K}+\left\|\frac{\partial f_{P}(z)}{\partial z}-\frac{\partial f(z)}{\partial z}\right\|_{K}+\left\|\frac{\partial f_{P}(z)}{\partial \bar{z}}\right\|_{K} \rightarrow 0 \text { as } \epsilon \rightarrow 0
$$

The rate of convergence depends only on $k_{0}, K$ and $\Omega$.

The rest of the paper will be organized into two sections which can be read independently. In the first section, we will explain how to generalize the method of [R-S] to obtain uniform convergence for $f_{P}$; here we work with the additional hypothesis that the ratios of radii of circles of $P$ are uniformly bounded. Note that the hypothesis of uniformly bounded radii ratios implies that the valences of $P$ are also uniformly bounded. In the second section we show how to modify the argument of [He] to prove Theorem 2.1.

Acknowledgement. The first named author wishes to thank Professor Jim Cannon for a discussion which helped him to clarify a misunderstanding.

\section{Convergence in the Case of Bounded Radil Ratio}

Let us first start with the following easy case; and see how the method of [R-S] can be extended.

Theorem 1.1. Assume that there is some fixed constant $M \geq 1$ so that the ratio of the radii of any two circles in $P$ is bounded by $M$. Then in any compact subset $K \subseteq \Omega$,

$$
\left\|f_{P}-f\right\|_{K} \rightarrow 0 \text { as } \epsilon \rightarrow 0
$$

Recall that the proof of [R-S] uses a compactness property of circle packings, a length-area inequality, and an approximate rigidity result for large pieces of the hexagonal packing. Let us start with the compactness property.

Lemma 1.2 (Compactness Property). Let $P_{n}$ be a sequence of circle packings with uniformly bounded valences, and let $c_{0, n}$ be a circle of $P_{n}$. Suppose that: 
(1) there is a sequence $k_{n}$ of positive integers with $\lim k_{n}=\infty$, such that for each $n$, the first $k_{n}$ generations of $P_{n}$ around $c_{0, n}$ is the 1-skeleton of a triangulation of the 2-disk, with circles in the first $k_{n}-1$ generations corresponding to the interior vertices of the triangulation;

(2) the centers of $c_{o, n}$ form a bounded subset of points and their radii are uniformly bounded both from above and from below.

Then a subsequence of $P_{n}$ converges to a circle packing $P_{\infty}$ in the plane whose carrier is a simply connected domain.

Proof. First choose a subsequence of $c_{0, n}$ which converges to a circle, say $c_{0, \infty}$. Now, since the valences of $P_{n}$ are uniformly bounded, we may choose a further subsequence such that the valences of $c_{0, n}$ in $P_{n}$ are the same, say equal to $j_{1}$. Let $c_{i, n}, i=1,2, \ldots, j_{1}$, be the circles of $P_{n}$ which are tangent to $c_{0, n}$. Then by the ring lemma $[\mathrm{R}-\mathrm{S}]$, the radii of these circles are also uniformly bounded both from above and from below. So, we may select a further subsequence such that for each $i=1,2, \ldots, j_{1}$, the sequence $c_{i, n}$ converges to some circle $c_{i, \infty}$. Because of the bounded valence property, the number of circles in any finite number of generations of $P_{n}$ arround $c_{0, n}$ is uniformly bounded, so we may continue this process for all generations to get a sequence of subsequences. Then the diagonal sequence will satisfy the requirement of the lemma.

In [R-S], a length-area argument was used to show that the border circles of $P^{\prime}$ shrink to points, and consequently the Caratheodory distance $d\left(\partial \Omega_{p}, \partial \Omega\right)$ tends to zero, as $\epsilon \rightarrow 0$. Those arguments extend to nonhexagonal packings in the following way.

Lemma 1.3 (Length-Area Result). Let $P$ be a circle packing in $\Omega$ such that the mesh of $P$ and Caratheodory distance $d\left(\partial \Omega_{p}, \partial \Omega\right)$ are bounded above by $\epsilon$, and the ratios of the radii of any two circles of $P$ are bounded above by $M$. Let $P^{\prime}$ be the isomorphic circle packing of $\mathbb{D}$ such that a circle $c_{0}$ in $P$ nearest to $z_{0}$ corresponds to a circle $c_{0}^{\prime}$ in $P^{\prime}$ centered at the origin. Then every border circle of $P^{\prime}$ has diameter less than $C M / \log (1 / \epsilon)$, where $C$ depends only on $\operatorname{dist}\left(z_{0}, \partial \Omega\right)$. 
Proof. Consider a border circle $c$ of $P$ and crosscuts of $\Omega$ centered at the center of $c$ with radii $5 k \epsilon,\left(k=1,2, \ldots, k_{\max }\right)$, where $k_{\max }=\left[\operatorname{dist}\left(z_{0}, \partial \Omega\right) / 5 \epsilon\right]-2$. The crosscut of radius $5 k \epsilon$ meets a number of circles of $P$; select from these a chain $S_{k}$ of tangent circles which separates $c$ from $c_{0}$ and which has a border circle at each end. The chains $S_{1}, S_{2}, \ldots$ so obtained will be disjoint. Note that if $m$ disks of radius $\geq r$ with disjoint interiors intersect a circle of radius $\rho$ then $m \leq 4 \rho / r$ (since, if the disks had radius exactly $r$, the area of the annulus of radii $\rho \pm r$ would be an upper bound for $m \pi r^{2}$ ). Therefore, the combinatorial length of $S_{k}$ is $\leq 20 k M$. By the Length-Area Lemma [R-S], the circle $c^{\prime}$ in $P^{\prime}$ which corresponds to $c$ has radius at most

$$
\left\{\sum_{k=1}^{k_{\max }}\left(\frac{1}{20 k M}\right)\right\}^{-1} \leq \frac{C M}{\log \frac{1}{\epsilon}} .
$$

Corollary 3.3 in [Schwarz, Lemma II] makes use of a length-area argument incorporating the isoperimetric inequality, and arrives at a much stronger conclusion. Its proof also extends, mutatis mutandi, to nonhexagonal packings and gives the following result.

Lemma 1.4 (Length-Area-Isoperimetric Inequality). With the notations and hypotheses of Lemma 1.3, every border circle of $P^{\prime}$ has diameter less than $C M \epsilon^{\pi^{2} / 80 M}$ where $C$ depends only on $d\left(z_{0}, \partial \Omega\right)$.

Proof of Theorem 1.1. Let $P_{n}$ be a sequence of circle packings satisfying the hypothesis of Theorem 1.1 such that $\epsilon_{n}=\max \left\{d\left(\partial \Omega_{P}, \partial \Omega\right)\right.$, mesh $\left.m(P)\right\}$ converges to 0 . Let $f_{P_{n}}: \Omega_{P_{n}} \rightarrow D$ be the associated mappings constructed in the introduction. We need to show that $f_{P_{n}}$ converges to the Riemann mapping $f: \Omega \rightarrow D$ which satisfies (0.2).

By the ring lemma of [R-S], $f_{P_{n}}$ are all uniformly quasiconformal mappings; and thus a subsequence (still denoted by the same notation) converges to some mapping, say $f^{\prime}: \Omega \rightarrow D$ which is either quasiconformal or a constant. But Lemma 1.3 implies the image of $f^{\prime}$ is $D$ and thus $f^{\prime}$ is a quasiconformal homeomorphism of $\Omega$ onto $D$. Clearly $f^{\prime}$ satisfies $(0.2)$.

It remains to show that $f^{\prime}$ is conformal. Let $\mu_{n}$ be the complex dilatation of $f_{P_{n}}$. We claim that $\mu_{n}$ converges to zero at almost every point of $\Omega$. Suppose 
by contradiction that there are a set of $w \in \Omega$ of positive measure, a $\delta>0$, and a subsequence (still denoted by $\mu_{n}$ ) of $\mu_{n}$ such that

$$
\left|\mu_{n}(w)\right| \geq \delta .
$$

From the definition of the mappings $f_{P_{n}}$, it follows that for each $n$ there is a triple of mutually tangent circles $c_{0, n}, c_{1, n}$ and $c_{2, n}$ of $P_{n}$ such that:

(i) $w$ is contained in the triangle of centers of $c_{0, n}, c_{1, n}$ and $c_{2, n}$;

(ii) if $c_{0, n}^{\prime}, c_{1, n}^{\prime}$ and $c_{2, n}^{\prime}$ are the corresponding circles of $P_{n}^{\prime}$ (constructed in the introduction), then the triangle of centers of $c_{0, n}^{\prime}, c_{1, n}^{\prime}$ and $c_{2, n}^{\prime}$ is not nearly similar to the triangle of centers of $c_{0, n}, c_{1, n}$ and $c_{2, n}$.

Clearly, the packings $P_{n}$ and $c_{0, n}$, and $P_{n}^{\prime}$ and $c_{0,1}^{\prime}$ satisfy the conditions of Lemma 1.2 after transformations by affine similarities. It follows that (some subsequence) of the transformed packings $P_{n}$ and $P_{n}^{\prime}$ converge to some infinite packings, say, $P_{\infty}$ and $P_{\infty}^{\prime}$. Since the circles of $P_{n}$ have uniformly bounded ratios, the carrier of $P_{\infty}$ must fill up the whole plane. Then by the rigidity result of [R-S], $P_{\infty}$ and $P_{\infty}^{\prime}$ are similar. This contradicts the above conclusion that the triangle of centers of $c_{0, n}^{\prime}, c_{1, n}^{\prime}$ and $c_{2, n}^{\prime}$ is not nearly similar to the triangle of centers of $c_{0, n}, \dot{c}_{1, n}$ and $c_{2, n}$. Hence $\mu_{n}(w)$ must converge to zero.

The rest of the proof is just the same as [R-S].

\section{Convergence in the Case of Bounded Valence}

In this section, we will prove the following stronger result under the weaker condition of bounded valence.

Theorem 2.1. Assume that the valences of $P$ are bounded by a positive integer $k_{0}$. Let $K \subseteq \Omega$ be a compact subset. Then

$$
\left\|f_{P}-f\right\|_{K}+\left\|\frac{\partial f_{P}(z)}{\partial z}-\frac{\partial f(z)}{\partial z}\right\|_{K}+\left\|\frac{\partial f_{P}(z)}{\partial \bar{z}}\right\|_{K} \rightarrow 0 \text { as } \epsilon \rightarrow 0 .
$$

The rate of convergence depends only on $k_{0}, K$ and $\Omega$.

We will begin with the definition of some constants similar to the hexagonal circle packing constants $s_{n}$ introduced in [R-S]. Let $n$ be an integer $\geq 2$. Suppose that $P_{n}$ is a circle packing in $\mathbb{C}$ such that

(1) The valence of $P_{n}$ is bounded by $k_{0}$; 
(2) The radii of the circles of $P_{n}$ are all bounded from above by some positive $r$; and there is some "center" circle $c_{0}$ of $P_{n}$ such that carrier of $P_{n}$ contains a closed disk of radius $(2 n+1) r$ which is concentric with $c_{0}$.

Here the carrier of a circle packing is understood to be the union of all the closed disks and the triangular interstices bounded by the circles.

Let $P_{n}^{\prime}$ be any other circle packing of $\mathbb{C}$ combinationally equivalent to $P_{n}$. Suppose that $c_{0}$ is surrounded by $c_{1}, c_{2}, \cdots, c_{k}$ in $P_{n}$; and $c_{0}^{\prime}, c_{1}^{\prime}, c_{2}^{\prime}, \cdots, c_{k}^{\prime}$ are the circles in $P_{n}^{\prime}$ corresponding to $c_{0}, c_{1}, c_{2}, \cdots, c_{k}$ respectively. Let

$$
d_{1}\left(P_{n}, P_{n}^{\prime}\right)=\max \left\{\frac{\operatorname{Radius}\left(c_{j}^{\prime}\right) / \operatorname{Radius}\left(c_{l}^{\prime}\right)}{\operatorname{Radius}\left(c_{j}\right) / \operatorname{Radius}\left(c_{l}\right)} ; 0 \leq j, l \leq k\right\}
$$

and let

$$
s\left(P_{n}\right)=\sup _{P_{n}^{\prime}}\left[d_{1}\left(P_{n}, P_{n}^{\prime}\right)-1\right]
$$

The following theorem follows mutatis mutandi from [He]. Actually the proof under the new setting is more direct.

Theorem 2.2. There is a constant $C$ depending only on $k_{0}$ such that

$$
s\left(P_{n}\right) \leq \frac{C}{n} .
$$

Proof. Let us normalize $P_{n}$ so that $c_{0}$ becomes the unit circle $\partial D=\{z \in$ $\mathbb{C} ;|z|=1\}$. Let $m$ be a positive integer with $m<n$. For $m=1$, let $P_{1}$ be the first generation of $P_{n}$ arround $c_{0}$; for $m>1$, let $P_{m}$ be the subpacking of $P_{n}$ which consists of all circles which are contained in the closed disk of radius $(2 m+1) r$ centered at the origin. Let $G_{m}$ be the Schottky group generated by inversions on the circles of $P_{m}$. Denote by $J_{m}$ the union of images by the transformations of $G_{m}$ of the interstices bounded by circles in $P_{m}$. We have the following area estimate.

Lemma 2.3. For any Möbius transformation $h$ preserving the unit disk $D$, we have

$$
\left|D \backslash h\left(J_{m}\right)\right| \leq C_{3} / m^{2}
$$

where $C_{3}$ depends only on $k_{0}$. 
Proof. The proof follows by the inductive argument of Lemma 3.1 of [He] with the following modifications.

(a) Since $P_{1}$ is the first generation of $P_{n}$ arround $c_{0}$ and the valence of $P_{n}$ is bounded by $k_{0}$, Lemma 3.2 of [He] also holds for $J_{1}$ defined here; where $\delta_{2}$ and $C_{3}$ denote some positive constants depending only on $k_{0}$.

(b) $H_{1}, H_{2}, \cdots, H_{n}$ in [He] should be replaced by $P_{1}, P_{2}, \cdots, P_{n}$. The other notations need no changes. For example, $U_{1}$ is the complement in $\hat{C}$ of the union of interstices and disks bounded by circles in $P_{1}$, etc.

(c) The last paragraph of page 405 should be replaced by:

Let $\Delta$ be a disk bounded by some circle $c=\partial \Delta$ of $P_{k} \backslash P_{k-1}, 2 \leq$ $k \leq l-1$, and let $z_{\Delta}$ be its center. Since the closed disk of radius $(2(l-k)+1) r$ centered at the center of $c$ is contained in the closed disk of radius $(2 l+1) r$ centered at the center of $c_{0}$, we have by inductive hypothesis,

$$
\left|\gamma\left(\Delta \backslash J_{l}\right)\right| \leq \frac{C_{3}}{\pi(l-k)^{2}}|\gamma(\Delta)| .
$$

Lines 4-11 of page 406 in [He] should be replaced by:

If $z \in \Delta, \partial \Delta \in P_{k} \backslash P_{k-1}, 2 \leq k \leq l-1$, then $|z| \geq r k \geq k$, since

$$
r \geq \operatorname{radius}(D)=1 \text {. }
$$

Thus

$$
\frac{C_{3}}{\pi(l-k)^{2}} \leq \frac{C_{3}}{\pi\left[(l-|z|)^{+}\right]^{2}}
$$

Let $\rho: U \longrightarrow[0,1]$ be the following function

$$
\rho(z)=\rho(|z|)=\min \left(\frac{C_{3}}{\pi\left[(\ell-|z|)^{+}\right]^{2}}, 1\right) .
$$

Then $\eta \leq \rho$ on $U_{1}$, and hence $\eta_{\gamma} \leq \eta \circ \gamma^{-1} \leq \rho \circ \gamma^{-1}(z)$. By the Ring lemma, the sizes of the circles in $P_{1}$ are uniformly bounded, so there is some $R>1$ depending only on $k_{0}$ such that $\{|z|>3 R\} \cup\{\infty\} \subseteq U_{1}$. Let $V=\{|z|>3 R\} \cup\{\infty\}$. Then $\cdots$.

(d) In lines 1 and 2 of page 407 in [He], we should let $\sigma_{1}=\frac{3 R}{z}$; and then line 3 should be replaced by 


$$
\begin{aligned}
& \frac{1}{|\gamma(V)|} \iint_{\gamma_{1}(V)} \rho \circ \gamma_{1}^{-1} d x d y \\
& =\frac{1}{\pi} \iint_{D} \min \left(\frac{C_{3}}{\pi\left[\left(\ell-\frac{3 R}{|z|}\right)^{2}\right]^{2}}, 1\right) d x d y \\
& \leq \frac{1}{\pi} \iiint_{\left.|z|<\frac{3 R}{\left(1-\sqrt{1-\delta_{2} / 2}\right) \ell}\right\}^{1 \cdot d x d y}} \\
& \quad+\frac{1}{\pi} \iint\left\{\frac{3 R}{\left(1-\sqrt{1-\delta_{2} / 2}\right) \ell} \leq|z| \leq 1\right\} \frac{C_{3} d x d y}{\pi\left[\left(\ell-\frac{3 R}{|z|}\right)^{2}\right]^{2}} \\
& \leq \frac{9 R^{2}}{\left(1-\sqrt{\left.1-\delta_{2} / 2\right)^{2} \ell^{2}}+\frac{1}{\pi^{2}} \iint_{D} \frac{C_{3} d x d y}{\left[1-\delta_{2} / 2\right] \ell^{2}}\right.}
\end{aligned}
$$

Similarly, (3.12) and (3.7) in [He] should be replaced by

$$
\frac{1}{|\gamma(V)|} \iint_{\gamma(V)} \rho \circ \gamma^{-1}(z) d x d y \leq \frac{9 R^{2}}{\left(1-\sqrt{1-\delta_{2} / 2}\right)^{2} \ell^{2}}+\frac{C_{3}}{\pi\left(1-\delta_{2} / 2\right) \ell^{2}}
$$

and

$$
\text { (3.7') } \quad \frac{\left|\gamma\left(U_{1} \backslash J_{\ell}\right)\right|}{\left|\gamma\left(U_{1}\right)\right|} \leq \frac{9 R^{2}}{\left(1-\sqrt{1-\delta_{2} / 2}\right)^{2} \ell^{2}}+\frac{C_{3}}{\pi\left(1-\delta_{2} / 2\right) \ell^{2}} .
$$

(e) Line (3.14) of [He] should be replaced by

$$
C_{3}=\max \left\{4 \pi, \frac{18 R^{2} \pi\left(1-\delta_{2}\right)\left(1-\delta_{2} / 2\right)}{\left(1-\sqrt{1-\delta_{2} / 2}\right)^{2} \delta_{2}}\right\}
$$

Proof of Theorem 2.2 (continued). The rest of the proof is identical to that of the estimate on $s_{n}$ in [He, §2].

Proof of Theorem 2.1. The proof is the same as in the case of hexagonal packings (see also [Ro]) except the following lemma which replaces the length-area argument. 
Lemma 2.4. Let $P$ be a circle packing in $\Omega$ such that the mesh of $P$ and Caratheo-dory distance $d\left(\partial \Omega_{p}, \partial \Omega\right)$ are bounded above by $\epsilon$, and the valence of $P$ is bounded above by $k_{0}$. Let $P^{\prime}$ be the isomorphic circle packing of $D$ and let $f_{P}: \Omega_{P} \rightarrow D$ be the mapping constructed in the introduction such that $f_{P}\left(z_{0}\right)=0$. Then the Caratheodory distance $d\left(\partial f_{P}\left(\Omega_{P}\right), \partial D\right)$ is less than or equal to $C \epsilon^{\alpha}$, where $C$ and $\alpha$ are some positive contants which depend only on $k_{0}$ and $d\left(z_{0}, \partial \Omega\right)$.

Proof. We need only show that every border circle of $P^{\prime}$ has diameter less than or equal to $C \epsilon^{\alpha}$. By the Ring Lemma of [R-S], $f_{P}$ is $C_{4}$-quasiconformal onto its image where $C_{4}$ depends only on $k_{0}$. Let $c$ be a border circle of $P$ and let $c^{\prime}$ be the corresponding border circle in $P^{\prime}$. We assume that $c$ is chosen so that $c^{\prime}$ has the biggest radius among the border circles of $P^{\prime}$; and we want to show that the radius of $c^{\prime}$ is bounded by $C \epsilon^{\alpha}$. Consider the annulus $A$ bounded by the circle $c$ and the circle $c_{1}$ concentric with $c$ which passes through $z_{0}$. Because the radius of $c$ is $\leq \epsilon$ and the radius $c_{1}$ is at least $\operatorname{dist}\left(z_{0}, \partial \Omega\right)$, the modulus of the annulus $A$ is at least $C_{5}|\log \epsilon|$. It follows that the extremal length between $c \cap \Omega_{P}$ and $c_{1} \cap \Omega_{P}$ in the domain $A \cap \Omega_{P}$ is at least $C_{5}|\log \epsilon|$.

Since $f_{P}$ is $C_{4}$-quasiconformal, the extremal length between $\gamma=f_{P}(c \cap$ $\left.\Omega_{P}\right)=c^{\prime} \cap f_{P}\left(\Omega_{P}\right)$ and $\gamma_{1}=f_{0}\left(c_{1} \cap \Omega_{P}\right)$ in $f_{P}\left(A \cap \Omega_{P}\right)$ is at least $C_{4} C_{5}|\log \epsilon|$. Since $f_{P}\left(z_{0}\right)=0$ and $z_{0} \in c_{1}, \gamma_{1}$ is a curve which separates the domain $f_{P}\left(\Omega_{P}\right)$ and which passes the origin 0 . On the other hand, because $c^{\prime}$ is a biggest border circle, the domain $f_{P}\left(\Omega_{P}\right)$ contains a cone neighbourhood of the center of $c^{\prime}$ of an angle bounded from below by some universal positive constant. It follows that the radius of $c^{\prime}$ is bounded from above by $C \epsilon^{\alpha}$, for some $\alpha>0$ and $C>0$. This completes the proof.

\section{REFERENCES}

[He] He, Z.X. An estimate for hexagonal circle packings, J. Differential Geom. 33 (1991), 395-412.

[Ro] Rodin, B. Schwarz's lemma for circle packings II, J. Differential Geom. 30 (1989), 539-554.

[R-S] Rodin, B. and Sullivan, D., The convergence of circle packings to the Riemann mapping, J. Differential Geom. 26 (1987), 349-360.

[Ste] Stephenson, K., Circle packings in the approximations of conformal mappings. Bull. Amer. Math. Soc. 23 (1990), 407-415. 
[Th] Thurston, W., The finite Riemann mapping theorem, International Symposium in Celebration of the Proof of the Bieberbach Conjecture, Purdue University, 1985.

Princeton University, U. S. A.

University of California at San Diego, U. S. A.

ReCeived April 8, 1992 Revised August 31, 1992 